\title{
A New Algorithm of Online Stator Faults Diagnosis of Three-Phase Induction Motors Using Duty Ratios of Half-Period Frequencies According to Phase Angle Changes
}

\author{
YoungJin Go ${ }^{1}$, Myoung-Hyun Song ${ }^{2}$, Jun-Young Kim², Buhm Lee ${ }^{3}$, Wangrim Choi ${ }^{4}$ and Kyoung-Min Kim ${ }^{3}$ \\ ${ }^{1}$ Dept. of Electrical Automation, Suncheon JEIL College, Deogwol-dong, Suncheon-city, Jeollanam-do, Republic of Korea \\ ${ }^{2}$ Dept. of Electric Control Eng., Sunchon National Univ., Maegok-dong, Suncheon-city, Jeollanam-do, Republic of Korea \\ ${ }^{3}$ Dept. of Electrical and Semiconductor Eng., Chonnam National Univ., Dundeok-dong, Yeosu-city, Jeollanam-do, Republic of \\ Korea \\ ${ }^{4}$ Dept. of Biomedical and Electronic Eng., Chonnam National Univ., Dundeok-dong, Yeosu-city, Jeollanam-do, Republic of Korea
}

\begin{abstract}
The causes of faults of induction motors are largely categorized into bearing fault, which causes a mechanical fault, and stator fault and rotor fault, which cause an electrical fault. A stator fault among these faults, which causes an electrical fault, occurs due to the breakdown of insulation, meaning the stator is directly connected with the power supply, and the direct connection is a direct cause of a major accident. For this reason, many studies are being performed to detect the faults. This paper explained the effects of a negative sequence on phase angle change by analyzing the effects of the existing negative sequence on the d-q transform of Park's vector approach. This paper suggested a new algorithm that identifies the causes of stator faults with the use of the change in the duty ratio of the half-period frequency of the frequency when a phase angle change occurs at that moment.
\end{abstract}

\section{Introduction}

Most of the faults of induction motors are caused by bearing fault (44\%); wrong winding or stator fault due to a stator winding short circuit fault or a stator winding open circuit fault $(26 \%)$; and fault of the bar of the rotor $(8 \%)$. The causes of induction motors are largely categorized into mechanical faults and electrical faults bearing faults causing mechanical faults; and stator faults and rotor faults causing electrical ones.

Among the causes, a stator fault occurs due to the breakdown of insulation of the stator, a deterioration in insulation of the stator winding is caused by various reasons, such as coil movement, thermal stress, overload, and mechanical vibration, and the deterioration in insulation of the winding results in a turn to turn short[1].

The occurrence of a turn to turn short causes a large circulating current to occur in the faulty loop, and the heat in the winding, which is proportional to the square of the stator current, plays a role in accelerating the occurrence of a more serious fault, such as phase-tophase short fault and a phase-earth fault, which cause a serious damage to the winding and core of the stator.

Hence this is the time when early diagnosis is desperately needed, as detecting a turn to turn short in the early stage can prevent a sequential damage to the motor and considerably reduce economic loss by minimizing the outage time of the motor, the number of additional workers, and the cost of repair.
Among methods of diagnosis of ITSC fault of induction motors, studies on MESA (motor electrical signature analysis) using only electrical signatures are being performed actively. [2] was researched through Park's vector (PV) approach, which is a representative one; as for [3], faults were identified by setting the circular distortion ratios at the threshold values with the use of DR; [4,5] were researched through AI technique; [6] was researched through wevelet analysis. As for [7,8], negative sequence currents were utilized; as for [9], negative sequence impedance was utilized. [10] was researched through a matrix of impedances modeling cross-coupling between positive and negative sequence components; and recently as for [11], studies were performed considering asymmetric elements in the machines in which a negative sequence current component is bound to occur.

But other papers, analysis of the stator fault, it is difficult to analyze online.

Typically, the line fault diagnosis method has been used in the PV.

This paper suggested a new fault diagnosis method in online using the duty ratio of half-period frequency according to phase angle change, with the consideration of the effects of negative sequence components on PV's d-q transform. 


\section{Background theory}

A three-phase induction motor can simplify MCSA by using Park's Vector Approach. The marks for a phasor space are located on the cross-section of an induction motor and are converted into arbitrary instantaneous values on the three-phase on the complex plane. The phasor space on this complex plane is rotated at the same angular rate as the angular frequency of the three-phase supply system.

Hence if the three-phase input current of an induction motor is applied to Park's Vector Approach, it is converted as follows (see the Eq. 1 and Eq. 2 below :

$$
\begin{aligned}
& I_{d}=\left(\frac{\sqrt{6}}{2}\right) i_{M} \sin (\omega t) \\
& I_{q}=\left(\frac{\sqrt{6}}{2}\right) i_{M} \sin \left(\omega t-\frac{\pi}{2}\right)
\end{aligned}
$$

here $i_{M}$ represents supply current's maximum value [A], $\omega$ represents supply angular frequency $(\mathrm{rad} / \mathrm{s})$, and $\mathrm{t}$ represents time parameter [s].
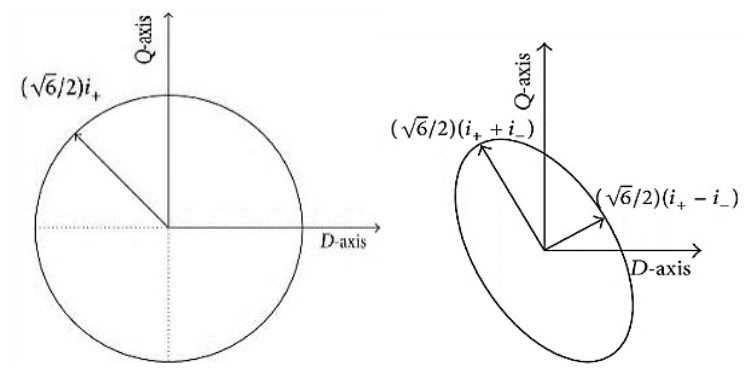

Figure 1. Pattern of Park's Vector

Fig. 1 shows the trace of a circle with its center at the origin on a coordinate plane. Under normal start conditions, the left pattern in Fig. 1 is shown. If a stator winding turn fault occurs, the right pattern (oval shape) in Fig. 1 is shown. More turn faults mean more asymmetric components of supply current, and more asymmetric components of supply current mean a higher degree of distortion of the trace of the pattern of Park's Vector[12].

The degree of distortion of the pattern has recently been defined as $D R$ (Distortion Ratio), which has been numerically calculated using the Eq. 3 and Eq. 4[3].

$$
\begin{gathered}
r=\sqrt{i_{d}^{2}+i_{q}^{2}} \\
D R=\frac{r_{\text {max }}}{r_{\min }}
\end{gathered}
$$

here $r$ represents the scalar of Park's Vector, $r_{\max }$ represents the broadest amplitude of it, and $r_{\min }$ represents the narrowest amplitude of it.

If the maximum value $r_{\max }$ is the same as the minimum value $r_{\text {min }}$, the induction motor is in the most ideal state $(D R: 1)$. As a larger difference between them means a higher $D R$, the phase current when the difference is large is under highly unbalanced conditions due to winding turn fault.

\subsection{Phase changes}

$r=\sqrt{I_{d}^{2}+I_{q}^{2}}$ represents the maximum value of current, actually determines the shape of a circle in Park's Vector Approach, and is an important factor representing the amplitude of current. Here the components $\cos \theta$ and $\sin \theta$ of $R_{e}$ and $I_{m}$ determine the position of $I_{s}$.

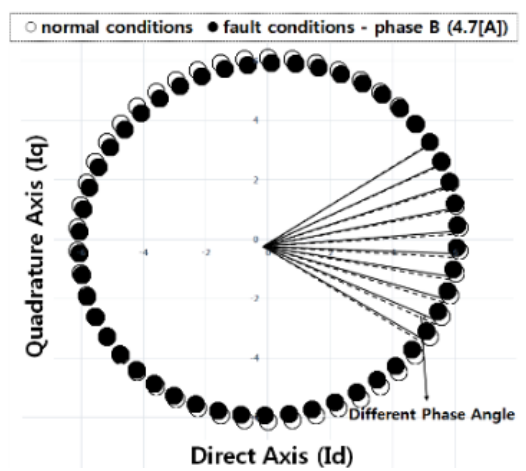

Figure 2. Different Phase Angle

When a fault occurred, a particular pattern was created by the actual phase angle $\theta$ although an analysis was performed only using the maximum value of $r$. This can be seen in Fig. 2.

As for Fig. 2 a simulation was performed based on a normal condition current of 5[A]. A current fault signal was generated in phase $\mathrm{B}$ among three phases (the phase current: 4.7[A]). If the difference in fault current is large, the change of the phase angle $\theta$ is also large.

To use the characteristics of the pattern according to the change of the phase angle $\theta$, the following Eq. 5 can be found using Euler's theorem[13].

$$
e^{j \theta}=\frac{I_{d}+j I_{q}}{r}=\frac{I_{d}+j I_{q}}{\sqrt{I_{d}^{2}+I_{q}^{2}}}
$$

\subsection{Negative sequence}

In steady state, the relationship between the vector operat or ' $a$ ' and the phase angle $\theta$ can be interpreted through a vector operator: This can be expressed as a theorem for th e operator ' $a$ ' as follows:

$$
\begin{aligned}
& a=1 \measuredangle 120^{\circ}=\cos 120^{\circ}+j \sin 120^{\circ}=-\frac{1}{2}+j \frac{\sqrt{3}}{2} \\
& a^{2}=1 \measuredangle 240^{\circ}=\cos 240^{\circ}+j \sin 240^{\circ}=-\frac{1}{2}-j \frac{\sqrt{3}}{2} \\
& a^{3}=1 \measuredangle 360^{\circ}=1 \\
& a+a^{2}=-\frac{1}{2}+j \frac{\sqrt{3}}{2}-\frac{1}{2}-j \frac{\sqrt{3}}{2}=-1 \\
& 1+a+a^{2}=0
\end{aligned}
$$

That is, in steady state, the total power of 3-phases must be zero.

Here a zero sequence component is not shown on a revolution indicator, and the component can be ignored. Therefore, when the total voltage of the positive sequence component of the stator is represented as $\tilde{V}_{1 s}$ and that of the negative sequence component is represented as $\tilde{V}_{2 s}$, it 
is possible to write the stator voltage of $\tilde{V}_{s}$ using the relationship $V_{d}+j V_{q}=\tilde{V}$ as follows:

$$
\widetilde{V}_{s}=\tilde{V}_{1 s}+\tilde{V}_{2 s}
$$

When seeing Eq. 11 based on d-q transform, it can be expressed as follows:

$$
v_{d q s}=\bar{V}_{1 d q s}^{e} e^{-j\left(\theta-w_{s} t\right)}+\bar{V}_{2 d q s}^{-e} e^{-j\left(\theta+w_{s} t\right)}
$$

here the formula $v_{d q s}=v_{d s}+j v_{q s}$ can be devised; it represents the positive sequence component of $\bar{V}_{1 d q s}^{e}=$ $\tilde{V}_{1 s}$ and the negative sequence component of $\bar{V}_{2 d q s}^{-e}=\tilde{V}_{2 s}^{*}$; and $t$ represents time and $\theta$ represents an angle of rotation.

In the Eq.12, the domain of $e$ is created by the positive sequence component that rotates together with the magnetic field and is expressed as such when only the positive sequence component in the 3-phase system exists; and $v_{d q s}$ equals $V_{1 s}$ and is expressed as $V_{1 d q s}^{e}$.

The domain of as $-\mathrm{e}$ is created by the negative sequence component that rotates together with the magnetic field and is expressed as such when only the negative sequence component in the 3-phase system exists; and $v_{d q s}$ equals $V_{2 s}^{*}$ and is expressed as $V_{2 d q s}^{-e}$.

These two domains rotate in the opposite direction to which the two pairs of frequencies of $d$-q transform rotate at the same speed.

This can be expressed as follows:

$$
\begin{gathered}
V_{1 d q s}^{e}=v_{1 d s}^{e}+j v_{1 q s}^{e}=\tilde{V}_{1 s} \\
V_{2 d q s}^{-e}=v_{2 d s}^{-e}+j v_{2 q s}^{-e}=\tilde{V}_{2 s} \\
V_{d q s}^{e}=v_{d s}^{e}+j v_{q s}^{e}=V_{1 d q s}^{e}+V_{2 d q s}^{-e} e^{-j 2 w_{s} t}
\end{gathered}
$$

In this way, it is also possible to infer currents and magnetic fluxes[14].

That is, it is possible to approach the interpretation of imbalance through the negative sequence component.

\subsection{Imbalance between interpreptation of imbalance}

frequencies

by

When supposing that the positive sequence component, the negative sequence component, and the zero sequence component of 3-phases exist as in these Figures,

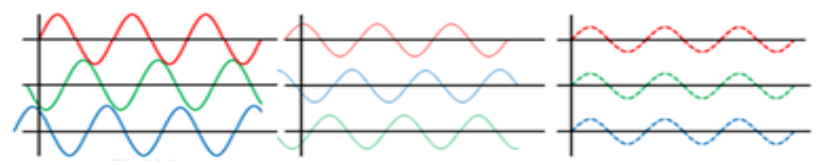

Figure 3. Example of 3 phase positive(L), negative(C), zero sequence component $(\mathrm{R})$

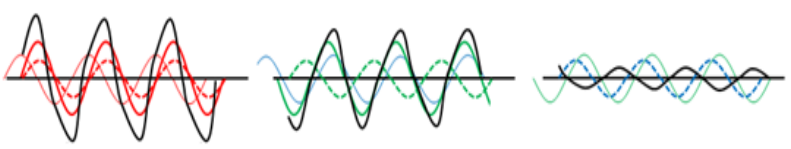

Figure 4. Sum of positive, negative and zero sequence component
As you can see in Fig. 4, an imbalance between frequencies occurs due to the negative sequence component, which causes $e^{-j 2 w_{s} t}$ to affect the total phase angle $\theta$ in the element $-e$, the domain of the negative sequence component in the Eq.15.

\subsection{An analysis of duty ratio of frequency}

This paper suggests a simplified algorithm of PWM (pulse width modulation) by detecting the rising times and falling times of half-period frequencies and analyzing distortions of the frequencies - the use of duty ratios of the frequencies for the analysis.

The amount of time for the half-period frequency at $60 \mathrm{~Hz}$ is approximately $0.0083 \mathrm{~s}$, and in steady state, the falling time must be the same as the rising time: approximately $0.04167 \mathrm{~s}$. But as you can see in Fig. 5, $0.0167 \mathrm{~s}$ (the total frequency: $60 \mathrm{~Hz}$ ) remains the same because of the negative sequence component; but there is a time difference between the rising time and falling time in the duty ratio of the half-period frequency.

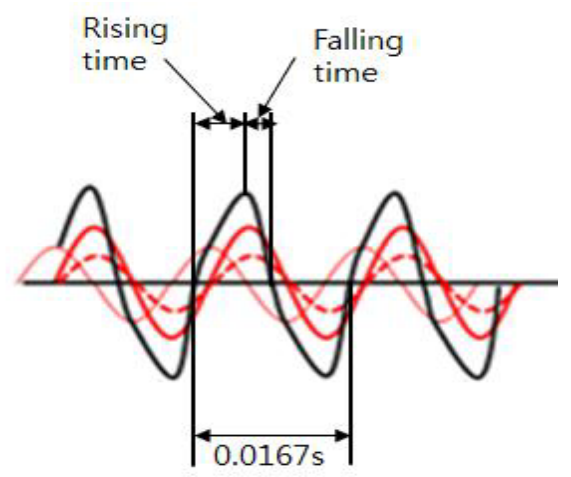

Figure 5. Half-period frequency duty ratio analysis algorithm

In addition, the value of the duty ratio represented a change in the falling time compared to the amount of time for the half-period frequency.

$$
\text { Duty Ratio[\%] }=\frac{\text { Falling time }}{\text { Rising time }+ \text { Falling time }} * 100 \%
$$

The measured frequency was expressed using Euler's theorem Eq. 5 and the duty ratio of the frequency in the formula above Eq. 16 was analyzed.

\section{Experiment}

\subsection{Experiment condition}

In this experiment, $1[\mathrm{HP}], 220[\mathrm{~V}], 4$ poles, $60 \mathrm{~Hz}$, a threephase induction was used.

The numbers of rotor slot and stator are 44, 36 .

In addition, the experiment was performed under the following conditions to consider changes in the load conditions.

- Operation of an induction motor by using inverter

- Adjustment of the motor operation speed by using dynamometer

- The initial speed - the rated speed $1690[\mathrm{rpm}]$ 
- The frequency of samplings, sampling rate and the No. of samplings: $1[\mathrm{~s}], 10,000[\mathrm{~S} / \mathrm{s}], 10,000[\mathrm{~s}]$

- Full load current and torque: $3.8[\mathrm{~A}] / 2.2[\mathrm{~A}]$ and $0.43[\mathrm{~kg} \mathrm{~m}]$

As an induction motor is operated by an inverter unlike a simulation, noise components exist as in the actual data in Fig. 6. For removal of noises, a filtering (IIR butterworth lowpass filter) was performed.
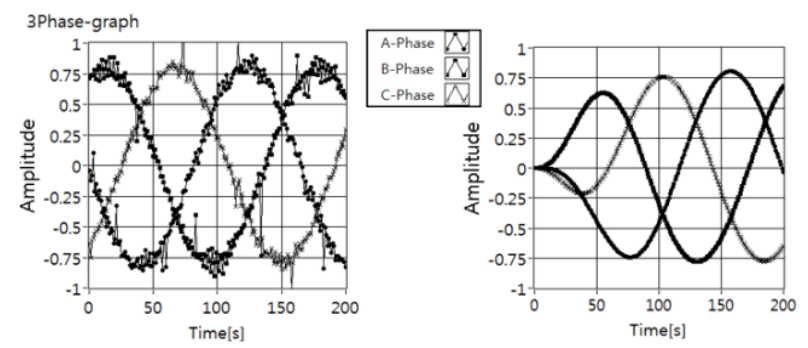

Figure 6. IIR filtering: before(L) and $\operatorname{after}(\mathrm{R})$

\subsection{Measurement error range}

At $60 \mathrm{~Hz}$ (power frequency), the amount of time for a period is approximately $0.0166-0.0167$ [s] and that for a half period is 0.00833 [s]. The ideal rising time or falling time should be measured as 0.00416-0.00417 [s], half of the amount of time for the half period.

To measure this, a sampling rate and synchronization must be achieved; for this paper, data collection was performed: 10,000 at the speed of 10,000 [S/s]. With the sampling rate used for the paper, the amount of time for one period was measured as $166-167\left[10^{-4} s\right]$. The frequency for the power should be accurately expressed with decimal points - analogue data - but the errors that occur due to the measured sampling rate should be considered as follows:

Table 1. Measurement error

\begin{tabular}{|l|c|c|}
\hline & One period (166) & One period(167) \\
\hline Half period & 83 & 83 or 84 \\
\hline Rising time & 41 or 42 & 42 \\
\hline Falling time & 42 or 41 & 42 \\
\hline
\end{tabular}

The maximum error range for a half-period is 83 $\left[10^{-4} s\right]$; and if the falling time is $41\left[10^{-4} \mathrm{~s}\right]$, as high as approximately $49.4 \%$ can be set as the error range for the normal duty ratio when there is no particular threshold setting method of the fault diagnosis.

\subsection{Frequency distortion phenomenon in case of stator fault and duty ratio}

An experiment was performed at $1690 \mathrm{rpm}$, the fullload state, to check the actual distortions of frequencies in steady state Fig. 7(L) and when an 8turn winding fault has occurred Fig. 7(R); and its result is as follows:
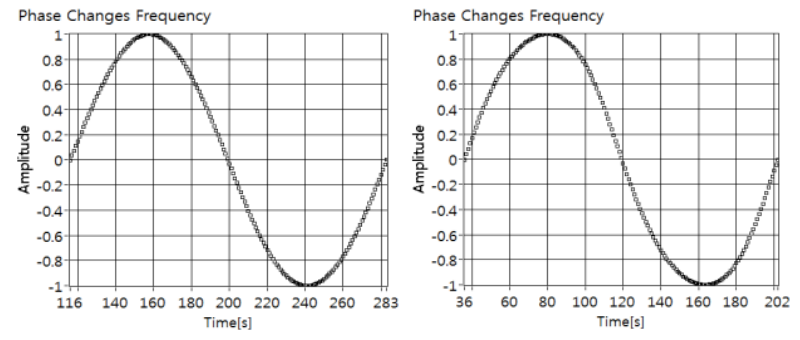

Figure 7. Frequency of 1690rpm by steady state(L), 8-turn windings $(\mathrm{R})$

The measurements were performed at the sampling rate of $10,000[\mathrm{~S} / \mathrm{s}]$, at frequency, the actual frequency of $60 \mathrm{~Hz}$ times 10,000. In Fig. 7, the X-axis represents time and $\left[10^{-4} s\right]$ represents the units. As for the sine wave in Fig. 7, it can be seen that the amount of time for one period, approximately $167\left[10^{-4} s\right]$ (from 116 to 283 $\left.\left[10^{-4} s\right]\right)$, is the same as that of one period at $60 \mathrm{~Hz}$, $0.0167[\mathrm{~s}]$.

As for these two frequencies, the result of a comparison of the rising times and falling times in the sections showing positive numbers is shown in Table 2 below:

Table 2. Average duty ratio at full-Load

\begin{tabular}{|l|l|l|l|}
\hline & rising time & falling time & Duty ratio[\%] \\
\hline Steady state & 41.7736 & 41.6038 & 49.8982 \\
\hline $\begin{array}{l}\text { 2-turn } \\
\text { winding }\end{array}$ & 41.9231 & 41.4615 & 49.7232 \\
\hline $\begin{array}{l}\text { 4-turn } \\
\text { winding }\end{array}$ & 42.6 & 40.88 & 48.9698 \\
\hline $\begin{array}{l}\text { 6-turn } \\
\text { winding }\end{array}$ & 43.2857 & 40.0816 & 48.0783 \\
\hline $\begin{array}{l}\text { 8-turn } \\
\text { winding }\end{array}$ & 44.3696 & 39.2391 & 46.9318 \\
\hline
\end{tabular}

The data in Table 2 are the result of the measurements performed at 1690rpm, the measurements being performed for only one second per each state, from steady state to 8-turn winding. The Table shows duty ratios and the data are the average figures for the falling times and falling times in the frequencies - the sections of positive numbers.

It can be seen that an increase in seriousness of turnto-turn short fault means an increase in rising time and a decrease in falling time.

Table 3. Average duty ratio at No-Load

\begin{tabular}{|l|c|c|c|}
\hline & rising time & falling time & Duty ratio[\%] \\
\hline Steady state & 41.7736 & 41.6226 & 49.9095 \\
\hline 2-turn winding & 42.4118 & 40.9804 & 49.1418 \\
\hline 4-turn winding & 43.8333 & 39.5208 & 47.4131 \\
\hline 6-turn winding & 45.4091 & 38 & 45.5568 \\
\hline 8-turn winding & 47.7179 & 35.7436 & 42.8265 \\
\hline
\end{tabular}

Table 3 shows the result of a comparison of the rising times and falling times and duty ratios for each turn to turn short fault at $1790 \mathrm{rpm}$ in no-load state. 
As the fault becomes more serious, the amount of rising time started to increase while that of falling time decreased, and it can be seen that there is to be a change in duty ratio.

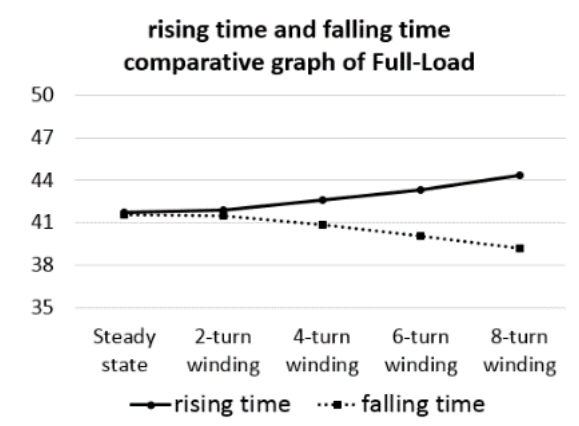

Figure 8. Rising time and falling time comparative graph of Full-Load

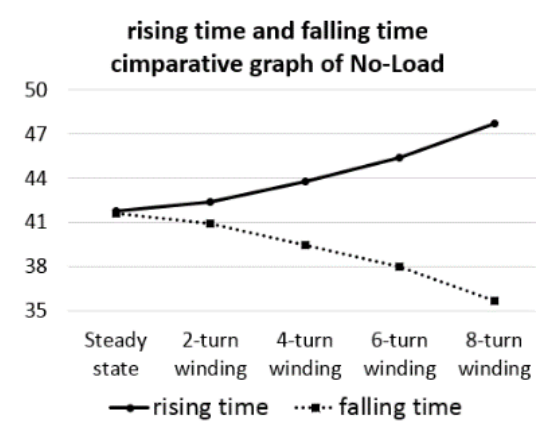

Figure 9. Rising time and falling time comparative graph of No-Load

Fig. 8 is a graph showing the result of a comparison of the rising times and falling times at full-load. Judging by each slope, it can be seen that there is no change in the overall frequency.

Fig. 9 is a graph showing the result of a comparison of the rising times and falling times at no-load. A pattern similar to that in Fig. 8 can be seen. Therefore, it can be seen that the duty ratio more dramatically changes as the fault becomes more serious at no-load, rather than fullload.

Fig. 10 is a graph showing the result of a comparison of duty ratios of average frequencies at full-load and noload.

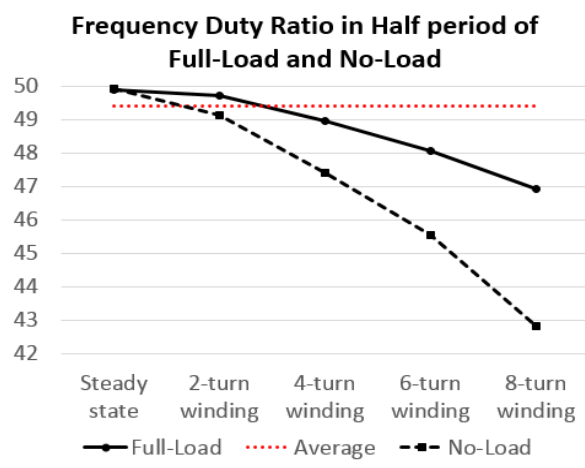

Figure 10. Frequency duty ratio of Full-Load and No-Load

When considering measurement error range, it is possible to judge accurately whether a 4-turn winding fault or a more serious turn-to-turn short fault has occurred although the ideal duty ratio $49.4 \%$ is considered as a reference threshold value (a 2-turn winding fault cannot be detected accurately in this case); and a 2-turn winding fault diagnosis can also be performed through the suggested method when being operated at no-load.

Fig. 11 below compares all of the data on stator fault.

The result of the preceding experiment was expressed as the sum of successive average figures for frequencies measured for one second. For the measurements for Fig. $11,10 \mathrm{rpm}$ was increased every 30 seconds, from 1690 rpm (full-load) to $1790 \mathrm{rpm}$, and the Fig. 11 shows the successive average figures for the duty ratios of the halfperiod frequencies measured every 30 seconds.

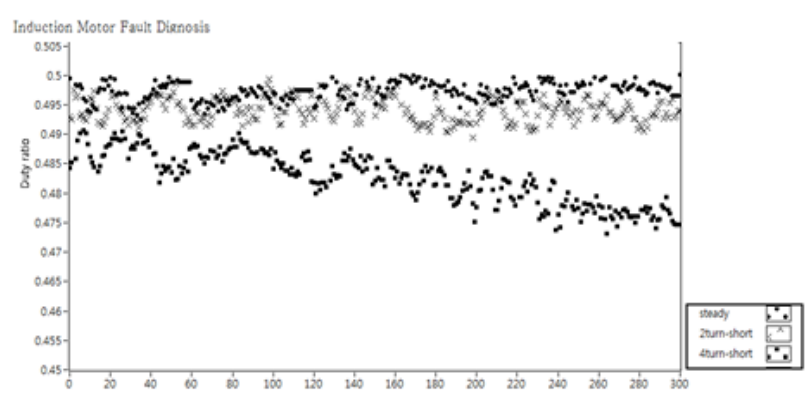

Figure 11. Result of induction motor fault diagnosis in electrical fault

In Fig. 11, the black circle represents steady state; the ' $\mathrm{X}$ ' mark 2-turn short fault; the quadrangle 4-turn short fault.

As for stator fault, it can be seen that linear separation is impossible at the ideal threshold value that the paper suggested when a 2-turn short fault has occurred; but linear separation from steady state is possible at the value when a 4-turn short fault has occurred.

\section{Conclusion}

The paper suggested a new technique that online stator faults with the use of the duty ratios of the half-period frequencies created when a phase angle change occurs, induction motor faults being caused by an electrical fault.

According to the findings of the paper, when a stator fault has occurred, the rising times of specific frequencies of the phase angle are longer. With these characteristics, which appear because of a phase angle change, now it is possible to judge swiftly whether a fault has occurred.

In addition, as for stator fault, it could be seen that linear separation from steady state is perfectly possible in case of the occurrence of a 4-turn short fault, complete fault state.

It is judged that analysis studies on the characteristics through long-term operation will need to be performed in the future, to find out a method of identifying 2-turn short faults, the situation where the fault has not become more serious yet.

\section{Acknowledgements}

This study was supported by the Ministry of Trade, Industry and Energy(MOTIE) through the Regional Innovation Centre Programme. 


\section{References}

1. S. Nandi and H. A. Toliyat., Record of the IEEE Industry Applications Conference, 197-204, (1999)

2. A. Ferrero and G. Superiti-Furga., IEEE Trans. Instrum. Meas 40, 10 (1991)

3. M. H. Song, K. N. Park, D. G. Han, and C. O. Yang., Trans. KIEE (in Korean) 14, 8 (2006)

4. F. Filippetti, G. Frauceschini, C. Tassoni and P. Vas, IEEE Trans. On Energy Conversion 21, 8 (2006)

5. R. M. Tallam, T. G. Habetler, R. G. Harley, O. J. Gritter, and B. H. Burton., Proceeding of IEEE IST Annual Meeting, 5 (2000)

6. M. A. Awadallah, M. M. Marcos, S. Gopalakrishman and T. W. Nehl., IEEE Trans. on Energy Conversion 21, 9 (2006)

7. G. B. Kliman, W. I. Premerlani, R. A. Koegl and D. Hoeweler., Electric Machines and Power Systems 28, $13(2000)$
8. R. M. Tallam, T. G. Habetler, and R. G. Harley., IEEE Tran. On Industry Applications 38, 6 (2002)

9. J. L. Kohler, J. Sottile, and F. C. Trutt, IEEE Trans. On Industry Applications 38, 6 (2002)

10. S. B. Lee, R. M. Tallam, and T. G. Habetler, IEEE Trans. On Power Electronics, 18, 8 (2003)

11. S. Bakhri, N. Ertugrul, and W. L. Soong., IECON $2012-38^{\text {th }}$ Annual Conference on IEEE Industrial Electronics Society, 6 (2012)

12. A. J. M. Cardoso, S. M. A. Cruz, and D. S. B. Fonseca., IEEE Trans. on Energy Conversion, 14, 4 (1999)

13. Y. J. Go, B. Lee, M. H. Song, K. M. Kim., Institute of Control, Robotics and Systems(in Korean) 20, 6 (2014)

14. R. Reginatoo and R. Bainy., IEEE PES General Meeting, 5 (2014) 